\title{
Correspondence
}

\section{Indications for cystourethrography in children with recurrent urinary infection}

Sir,

While fully agreeing with Shah et al. (Archives, 1978, 53, 210) that cystourethrography (CUG) is indicated in all children with recurrent urinary tract infection, it is less easy to accept that this should be done in all cases presenting under age 5 . In their case reports, 3 children presented at 5 years or under. In all these other factors would have led me to request CUG:

Case 11 Clinic attendances were irregular and there were recurrent infections.

Case 12 Was treated with continuous chemotherapy, which would have been the treatment of choice even if the reflux had been seen initially. One presumes there were no infections during these 2 years.

Case 14 This was the most disturbing case, but there was a minor abnormality on the IVU and some failure to thrive, both of which should have led to CUG.

Thus while I agree that one should have a high index of suspicion in the case presenting under the age of $5, \mathrm{I}$ feel that this paper, and others I have read, does not present a convincing case for routine use of this difficult and unpleasant examination in these children, provided regular and conscientious follow-up, including the use of dip slide urine testing at home, is possible.

\section{A. Porter \\ Kent and Canterbury Hospital, Canterbury, Kent CT1 3NG}

Dr Shah and co-authors comment:

The purpose of our case reports was to illustrate the reputedly rare development of new renal scars and not primarily to make a case for performing CUG routinely under 5 years of age. Nevertheless, it is a fact that 2 out of the 3 children less than 5 years old at presentation (Cases 12 and 13, not 11 as stated by Dr Porter) had completely normal IVUs in spite of grade III reflux. He must surely agree that the progression from a normal IVU to chronic renal failure in Case 13, in only 4 years, was both devastating and unexpected. Had this girl had a CUG performed initially, the outcome might have been more favourable.

Since most reinfections occur within a few weeks or months of discontinuing initial treatment of urinary tract infection, we agree that it is acceptable to defer CUG until first relapse, even under 5 years of age, provided that the urine is regularly cultured and that the IVU is completely normal. At this hospital, radiologists, nephrologists, and urologists meet weekly to review clinical records and $x$-rays of our own and referred patients and, not infrequently, we spot minor abnormalities which have been overlooked elsewhere.

However, clinical practice tends to be influenced by such variables as special interest and pressure of work, and we are unsure that the standards at which we and Dr Porter aim, are universally attainable. In less than perfect circumstances we believe that the risk that potentially damaging reflux, together with asymptomatic bacteriuria, may remain undetected for long periods probably outweighs the psychological disadvantages of the CUG examination.

Dr Porter refers to CUG as being difficult and unpleasant. As a regional referral centre we perform approximately 300 CUGs annually and rarely experience either technical difficulty or a severely disturbed child. This is largely attributable to skill and experience as well as to the sympathetic approach of nurses, radiographers, and others who devote their whole professional lives to the needs of children alone.

K. J. ShaH and R. H. R. White The Children's Hospital, Ladywood Middleway, Ladywood, Birmingham B16 8ET D. G. RoBINS St Luke's Hospital, Warren Road, Guildford, Surrey GU1 3NT

\section{Effect of heat on antimicrobial proteins in human milk}

Sir,

The interesting paper by Evans et al. (Archives, 1978, 53, 239) concludes that for human milk pasteurisation should be at $62^{\circ} \mathrm{C}$ for $30 \mathrm{~min}$, but that no commercial apparatus for this purpose is available in the United Kingdom.

In our study on human milk pasteurisation we described apparatus specifically designed for the accurate and uniform heat treatment of small volumes of human milk (Gibbs et al., 1977). This apparatus is available from Vickers Limited, Medical Engineering, Priestley Road, Basingstoke, Hants.

\section{J. D. BAUM \\ Department of Paediatrics, John Radcliffe Hospital, Headington, Oxford OX3 9EDU}

\section{Reference}

Gibbs, J. H., Fisher, C., Bhattacharya, S., Goddard, P., and Baum, J. D. (1977). Drip breast milk: its composition, collection and pasteurisation. Early Human Development, 1, 227-245. 